\title{
Reconfigurable and programmable origami dielectric elastomer actuators with 3D shape morphing and emissive architectures
}

\author{
Jiangxin Wang ${ }^{1}$, Shaohui $\mathrm{Li}^{1}$, Dace Gao ${ }^{1}$, Jiaqing Xiong ${ }^{1}$ and Pooi See Lee $\mathbb{B}^{1}$
}

\begin{abstract}
Soft actuators with the capability to generate programmable and reconfigurable motions without the use of complicated and rigid infrastructures are of great interest for the development of smart, interactive, and adaptive soft electronic systems. Here, we report a new strategy to achieve a transparent and reconfigurable actuator by using a dielectric elastomer actuator (DEA), which provides mechanical strains under electrical bias, integrated with origami ethyl cellulose (EC) paper that "instructs" the shape changes of the actuator. The actuator can be reconfigured and multiple mechanical motions can be programmed in the device by creating crease patterns that induce variations in the local stiffness to direct the actuations. With the versatile design and fabrication approach, a light emission device with dynamic shape changes was demonstrated.
\end{abstract}

\section{Introduction}

Reconfigurable shape changes on demand enable alterable mechanical functionalities for multiple task applications. Emerging progress has been made through the application of different materials and strategies ${ }^{1}$. For instance, modular self-reconfigurable robots can change their shapes by rearranging individual modules to adapt to different circumstances and tasks ${ }^{2}$. Liquid crystal polymer networks that respond to light or humidity stimuli can be reconfigured for different shape morphing by acidic patterning ${ }^{3}$, base treatment ${ }^{4}$, or photochemical program$\mathrm{ming}^{5}$. Among the varieties of soft actuators, the dielectric elastomer actuator (DEA) has been intensively investigated, with the benefits of scalable dimensions, simple architectures, excellent mechanical compliance, and large deformations $^{6-9}$, making it an ideal candidate for programmable soft robotic systems. However, conventional DEAs typically show predetermined shape changes. The development of a simple and programmable approach to achieve reconfigurable DEA devices with versatile, fast,

\footnotetext{
Correspondence: Pooi See Lee (pslee@ntu.edu.sg)

${ }^{1}$ School of Materials Science and Engineering, 50 Nanyang Avenue, Nanyang Technological University, Singapore 639798, Singapore
}

and reliable three-dimensional (3D) actuations is still lacking.

The mechanism of DEAs is based on the Maxwell stress $\left(P=\varepsilon_{r} \varepsilon_{0} E^{2}\right.$, where $P$ is the generated pressure, $\varepsilon_{0}$ and $\varepsilon_{\mathrm{r}}$ are the dielectric constant of vacuum and the relative permittivity of the dielectric, and $E$ is the electrical field) created on the elastic dielectric layer sandwiched between two compliant electrodes ${ }^{6,10}$, which induces in-plane strains in the devices. To achieve out-of-plane actuations from DEA devices, additional structure designs are required. For instance, the planar strains could be directed into out-of-plane displacement via integration with a gas chamber $^{11-13}$. Similarly, other fluids, such as water ${ }^{14}$ and silicone gel $^{15}$, were also applied to create pressurized chambers to control the actuation behaviors of DEA devices. Alternatively, DEAs with multiple device layers were embossed onto rigid frames, which confined the lateral expansion of the DEA devices and ensured the direction of the orthogonal displacement ${ }^{16,17}$. Unfortunately, these approaches require bulky fluid compression systems or rigid frames to direct and confine the actuation behaviors of the DEAs. To circumvent this difficulty, a unimorph structure with layers of different materials in 
the DEAs provides a simple and effective approach ${ }^{18-22}$. Representative applications were demonstrated, such as soft grippers, by incorporating rigid fibers into the DEAs ${ }^{19}$ and programmable multilayer $\mathrm{DEAs}^{20}$. A limitation remains in that the actuation behaviors of these devices cannot be modified from their original design after fabrication. The reconfigurability of DEAs will be addressed in this work.

Origami is a nature-inspired ancient art that can be used to enable variable $3 \mathrm{D}$ shape designs through the creation of crease patterns. It has been widely employed in paper art, engineering ${ }^{23-25}$, biological systems ${ }^{26,27}$, etc. Utilizing the origami approach, complex shapes and motions can be programmed in soft robotics. For instance, elastomeric artificial muscles were reported by using an origami skeletal structure with a negatively pressurized actuator ${ }^{28}$, soft pneumatic actuators with controllable motions were demonstrated by using stretchable elastomers and origami paper $^{29}$, and soft robots with a 3D origami structure were programmed by using a spatially defined shape memory polymer ${ }^{30}$. In this study, we demonstrate that by employing the origami principle, a programmable and reconfigurable transparent unimorph actuator can be achieved by integrating origami ethyl cellulose (EC) paper with DEAs. EC paper possesses the advantages of high transparency, excellent flexibility and foldability, and robust mechanical endurance ${ }^{31}$. Outof-plane and alterable shape changes can be programmed in the DEA devices by creating reconfigurable crease patterns. Benefitting from the simple and predictable approach, we also demonstrated an emissive actuator with programmable shape morphing by integrating an alternating current electroluminescent (ACEL) device with the reconfigurable actuator, featuring promising applications in camouflage, virtual displays, and other interactive soft electronic systems.

\section{Materials and methods}

\section{Fabrication of the EC paper and ionic conductor}

EC was dissolved in $N$-methyl-2-pyrrolidone (NMP) or dimethylformamide (DMF) at a concentration of $5 \%$ by weight. The prepared solution was casted onto a glass substrate and dried at $80^{\circ} \mathrm{C}$ for $10 \mathrm{~h}$. Afterward, transparent EC paper with a thickness of $50 \mu \mathrm{m}$ was achieved and peeled off from the substrate for device fabrication.

The ionic conductor was prepared following the same approach as in our previous report ${ }^{32}$. In brief, poly(methyl methacrylate) (PMMA, average $\mathrm{Mw}=\sim 350,000$ ), lithium perchlorate $\left(\mathrm{LiClO}_{4}\right)$, propylene carbonate (PC), and acetonitrile were purchased from Sigma-Aldrich and used as received. The weight ratio between $\mathrm{LiClO}_{4}, \mathrm{PC}$, acetonitrile, and PMMA was fixed at 1.5:4:16:3. $\mathrm{LiClO}_{4}$ was first dissolved in $2 \mathrm{~g}$ of PC. After the $\mathrm{LiClO}_{4}$ was fully dissolved, acetonitrile was mixed with the solution, followed by PMMA powder. The mixtures were stirred at $1000 \mathrm{rpm}$ overnight. A viscous and clear gel-like solution was obtained after the PMMA was fully dissolved.

\section{Fabrication of the transparent DEA devices}

$3 \mathrm{M}$ VHB tape (4905) with a thickness of $500 \mu \mathrm{m}$ was used as the dielectric layer. The ionic conductor was first applied onto both sides of the dielectric layer by stencil printing. After deposition, the ionic conductor was allowed to dry in atmosphere for 30 min to fully evaporate the acetonitrile. The ionic conductor had a thickness of $\sim 70 \mu \mathrm{m}$ after drying. The drying process is important to avoid damage to EC paper due to the presence of solvents such as acetonitrile. Afterward, the EC paper was attached to the dielectric layer with the strong adhesion force provided by the $3 \mathrm{M}$ VHB tape (peeling force of $2100 \mathrm{~N} / \mathrm{m}$, $3 \mathrm{M}$ VHB tape product information). The crease patterns were created by folding the EC paper layer at a bending angle of $180^{\circ}$ for $\sim 5 \mathrm{~min}$.

\section{Integration with the ACEL device}

Fabrication of the transparent DEA device was first carried out following the approach described above. ZnS: $\mathrm{Cu}$ microparticles were supplied by Shanghai KPT company. Pure PDMS was prepared by mixing the base and curing agent (Sylgard 184, Dow Corning) with a weight ratio of 10:1. $\mathrm{ZnS}: \mathrm{Cu}$ and the liquid PDMS were mixed in a weight ratio of $1: 1$. The liquid mixture was applied onto a glass slide by stencil printing. The deposited film was degassed and thermally cured at $60^{\circ} \mathrm{C}$ for $2 \mathrm{~h}$, resulting in an $\sim 200 \mu \mathrm{m}$ thick film after curing. The emission film was peeled off from the glass slide and transferred onto the top electrode of the fabricated DEA devices. Another ionic conductor layer was applied onto the emission layer to complete the device fabrication.

\section{Characterization}

A function generator (Yokogawa FG 300) was used to generate different waveforms, which were amplified by a power amplifier (Trek, Model PD05034). Emission from the device was collected by an optical fiber connected to an Acton SP-2300 monochromator. Emission spectra from the devices were measured by a Princeton Pixis 100B spectroscopy CCD detector on the monochromator. Displacement of the actuator was measured by a laser displacement sensor (optoNCDT 2300).

\section{Results}

\section{Mechanism and characterization of the actuator}

The transparent actuator consists of a simple architecture with a dielectric layer sandwiched between two transparent ionic conductor layers and an EC paper layer, as shown in Fig. 1a. Ionic conductors are used as electrodes with excellent transparency (transmittance can 

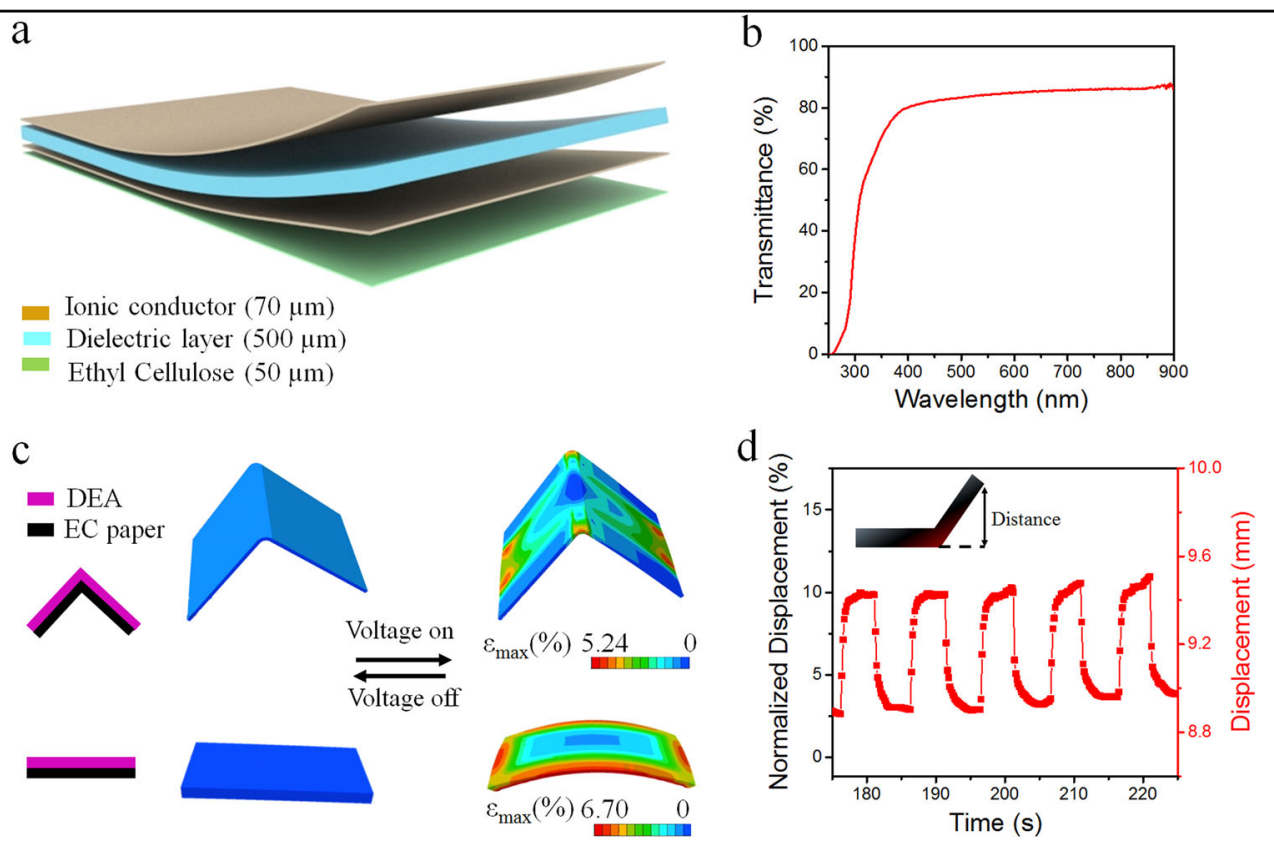

Fig. 1 The DEA device programmed with a crease pattern. a A schematic image showing the configuration of the DEA device. $\mathbf{b}$ The transmittance spectrum of the DEA device. $\mathbf{c}$ Strain distributions of the DEA device with and without a crease pattern obtained by FEA simulations. d The vertical displacement of the actuator under a square wave electrical bias. The inset shows a schematic of the device configuration under measurement.

reach $\sim 100 \%$ ) and mechanical compliance (stretchability can reach $>600 \%$ strain depending on the host polymer $)^{8,33}$. Under electrical bias, the voltage can be effectively coupled onto the dielectric layers due to the large capacitance of the external electrode/ionic conductor interface and the small capacitance of the dielectric layer, as illustrated by the schematic in Supplementary Fig. S1. A detailed discussion of the working mechanism can also be found in our earlier study ${ }^{32}$ or in previous reports ${ }^{33,34}$. With the excellent transparency of the ionic conductor, EC paper, and dielectric layer, the fabricated device achieved a transparency of $84 \%$ at $550 \mathrm{~nm}$, as shown in Fig. 1b. The high transparency provides an advantage for the integration of this actuator with other electronic devices that require light transmittance, such as lightemitting devices, electrochromic windows, and solar cells.

The actuator with a crease line created in the device was investigated first. The device showed a folding motion with respect to the crease line under electrical bias. Figure 1c shows the finite element analysis (FEA) simulation result of the actuator with (top image) and without (bottom image) the crease pattern. Under electrical bias, the Maxwell stress leads to biaxial tensile strains in the dielectric layers. Without the crease pattern, the unimorph structure demonstrates symmetric convex deformation due to the larger Young's modulus of the EC paper and relatively small Young's modulus of the elastic dielectric layer $\left(E_{\mathrm{EC} \text { paper }} / E_{\text {dielectric layer }}=3.3 \times 10^{3}\right.$ at $5 \%$ strain; the stress-strain curves of the EC paper and the elastic dielectric layer are provided in Supplementary Fig. S2). By introducing the crease line at the center of the device, the symmetric convex deformation is broken. Instead, the actuator shows symmetric folding with respect to the crease line due to the significantly increased stiffness encountered by the bending in the direction perpendicular to the crease line. A previous study reported a similar effect by embedding rigid fibers in unimorph DEA devices ${ }^{19}$. The approach of crease pattern creation in this study provides a versatile and reprogrammable process to control the actuation behavior on demand after device fabrication. As shown in the FEA simulation result (Fig. 1c, top image), the strains were distributed with a twofold symmetry with respect to the crease line under electrical bias. The device was recovered with the relaxation of the compression strain in the elastic dielectric layer and the relaxation of the bending strain in the EC paper when the electrical bias was switched off. A flapping motion could be easily programmed in the device by the creation of two crease lines (a square wave electrical bias, $50 \%$ duty cycle, $6 \mathrm{kV}, \sim 0.5 \mathrm{~Hz}$, was applied), as shown in Supplementary Movie S1.

To characterize the dynamic performances of the DEA device (length $\times$ width $=1.5 \times 1 \mathrm{~cm}$ ), half of the device was fixed onto a horizontal substrate. The device was actuated under electrical bias, with changes in the vertical displacement in the other portion of the device, as indicated 
in the Fig. 1d inset. The vertical actuation at the free end of the device was measured by a laser displacement sensor. As shown in Fig. 1d, the DEA device showed a stable and repetitive vertical displacement of $\sim 0.5 \mathrm{~mm}(\sim 7 \%$ of the device length allowed to undergo free motion) with the applied square wave bias $(50 \%$ duty cycle, $4 \mathrm{kV}$, $0.1 \mathrm{~Hz}$ ). The blocked force from the DEA device under different voltages is provided in Supplementary Fig. S3. The blocked force reached $2 \mathrm{mN}$ at a voltage of $5 \mathrm{kV}$, which was in a similar range as the unimorph DEA device with multilayers $(5 \mathrm{mN}$ and $17.5 \mathrm{mN}$ with 3 DEA layers and 7 DEA layers, respectively, at $5 \mathrm{kV})^{20}$. The relatively small blocked force from our device could be attributed to the single layer structure and the additional force required to deform the EC paper with a larger Young's modulus under actuation. The mechanical stability of the device was also studied and is provided in Supplementary Fig. S4.

\section{Reconfigurable motions and programmable 3D morphing with the actuator}

The actuation behavior of the device could be reconfigured by changing the crease patterns in the EC paper, which was used as the skeletal structure in the DEA device to control the actuation behavior. By changing the crease patterns, the device demonstrated variable localized mechanical stiffness, leading to different actuation behaviors. With the origami process, different ways to create the crease patterns could be explored to reconfigure the device actuations, as schematically illustrated in the Supplementary Information (Fig. S5). This provides a high degree of freedom in reconfiguring device actuation with the origami process. As an illustrative example, we first fabricated a device with two crease lines (the crease patterns were created with the EC paper layer folded inward, as shown in the inset of Fig. 2a). The device showed a folding motion under electrical bias, as shown in Fig. 2a. The folding angle (as indicated in the inset of Fig. 2a) of the actuator decreased from $127^{\circ}$ to $103^{\circ}$ as the applied voltage increased from 0 to $6.5 \mathrm{kV}$, as shown in Fig. 2b. Subsequently, the crease pattern in the device was reconfigured with the dielectric layer folded outward (as indicated in the inset of Fig. 2c). With the reconfigured crease pattern, the device showed an unfolding motion under electrical bias. The folding angle (as indicated in the inset of Fig. 2c) of the actuator changed from $119^{\circ}$ to $127^{\circ}$ with increasing applied voltage from 0 to $6 \mathrm{kV}$, as shown in Fig. 2d. Changes in the folding angles under actuation could be further improved by using dielectric materials with larger dielectric constants or by using multiple DEA layers.

The DEA devices were programmed to achieve more complex 3D motions. Bidirectional folding of the device was achieved with two DEA devices fabricated on opposite sides of the EC paper. Crease patterns were created
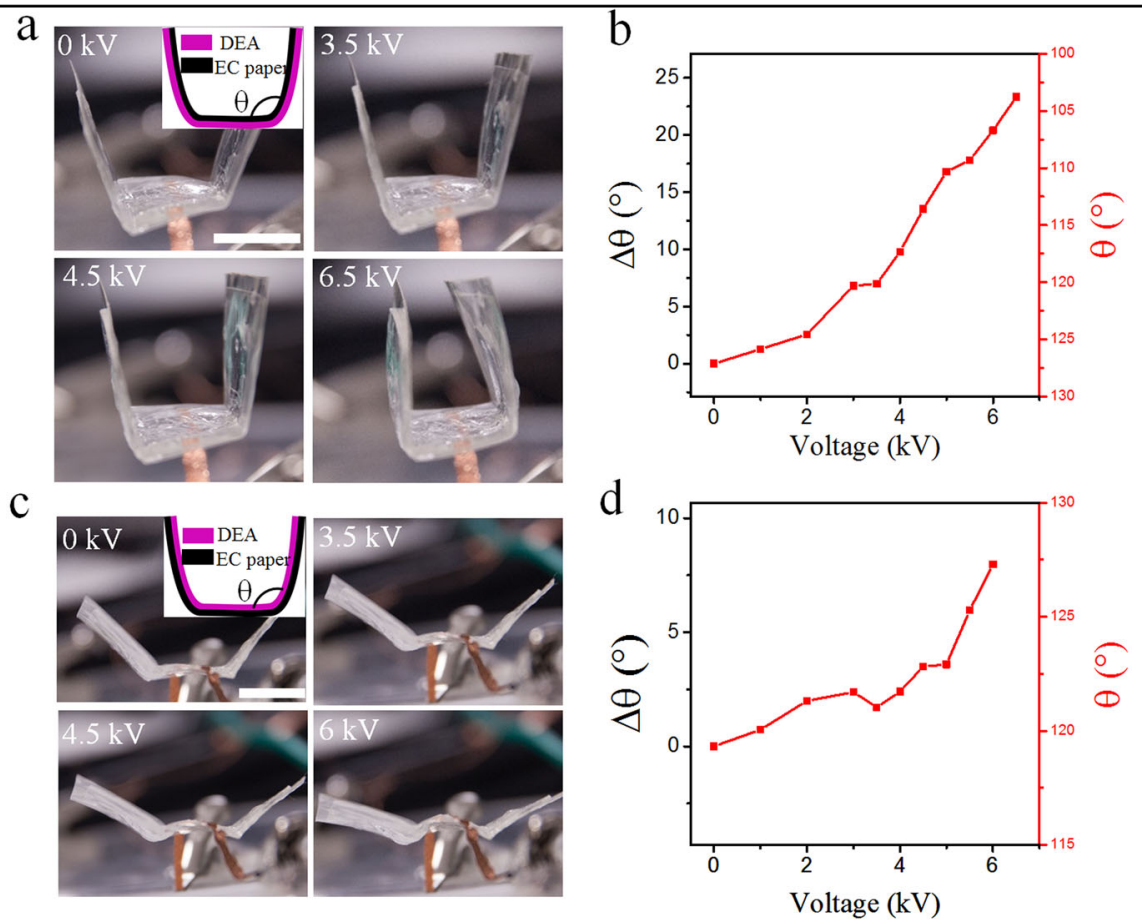

Fig. 2 The reconfigurable DEA device showing folding and unfolding morphing. a Photographs showing folding of the DEA device under an increased electrical bias. $\mathbf{b}$ Change in the folding angle under different electrical biases. c Photographs showing the unfolding of the DEA device under an increased electrical bias. $\mathbf{d}$ Change in the folding angle under different electrical biases. The scale bar in the photographs is $10 \mathrm{~mm}$. 
with the EC paper folded inward at the two ends of the device. The FEA prediction and corresponding experimental results are provided in Fig. 3a. The top images show the device in the off state. The device showed downward and upward folding at the left and right ends of the device, respectively, as indicated in the bottom images of Fig. 3a. Consistent actuation behaviors were observed between the FEA prediction and experimental results. The dynamic actuation process of the device is provided in Supplementary Movie S2. A self-folding origami box could also be fabricated by creating four crease patterns (with the EC paper folded inward) in the device. The configuration of the device is shown in Fig. 3b. The good agreement between the experimental results and the FEA predictions provides further evidence that the simple and predictable approach is effective in achieving programmable actuation behavior in DEA devices. Movie S3 is provided in the supplementary information, which shows the dynamic actuation process of the device along with the FEA predictions and experimental results.

\section{An emissive flapping device obtained by integrating the ACEL device with the actuator}

Light-emitting devices with dynamic morphing that can provide graphic displays and shape changes enable interesting applications in volumetric displays, interactive human-machine interfaces, biological systems, and camouflage electronic skins ${ }^{35-37}$. To illustrate the potential of the approach, we demonstrated an emissive flapping device by integrating the ACEL device with the programmable DEA device, mimicking a firefly that can glow to interact and communicate with other species ${ }^{38}$. Figure 4a shows the architecture of the integrated device. A common electrode was shared between the DEA device and the ACEL device. The cross-section view and equivalent circuit of the integrated device are provided in Supplementary Fig. S6. Electrical biases were independently applied to the DEA device and the ACEL device. Ionic conductors were used as the electrode for the ACEL device. Similar to the working mechanism in the DEA device, the electrical bias can be effectively coupled to the emissive layer due to its significantly smaller capacitance compared to the capacitance of the external electrode/ ionic conductor interface ${ }^{32,39,40}$. A photograph of the integrated device with light emission is provided in Fig. 4b. The ACEL device remained functional in the integrated device under dynamic shape morphing, as shown in the video provided in Supplementary Movie S4. For the emissive flapping device, a constant AC bias $(300 \mathrm{~V}$, $50 \mathrm{~Hz}$ ) was applied on the ACEL device, and a square wave electrical bias $(50 \%$ duty cycle, $5 \mathrm{kV})$ was applied on the DEA device. The frequencies of the square wave were ramped up from $1 \mathrm{~Hz}$ to $20 \mathrm{~Hz}$. A reduced flapping amplitude was observed under increasing frequency, which could be attributed to the viscoelastic response of the dielectric elastomer. Emission was maintained in the ACEL device under the flapping motion. The emission spectrum of the ACEL device is provided in Fig. 4c, which was centered at $\sim 500 \mathrm{~nm}$ with a FWHM (full-width-athalf-maximum) of $\sim 70 \mathrm{~nm}$. Different emission colors could also be achieved with different active dopants or dopant concentrations in the phosphors ${ }^{35}$. Figure $4 \mathrm{~d}$ shows a repetitive vertical displacement of $\sim 0.68 \mathrm{~mm}$ in the device under a square wave electrical bias $(50 \%$ duty cycle, $5 \mathrm{kV}, 1 \mathrm{~Hz}$ ). The demonstrated device provides a new approach to integrate reconfigurable 3D morphing structures with elastic light-emitting devices, which enables active shape morphing and color changes for interactive electronic interfaces, smart soft robots, and volumetric displays.
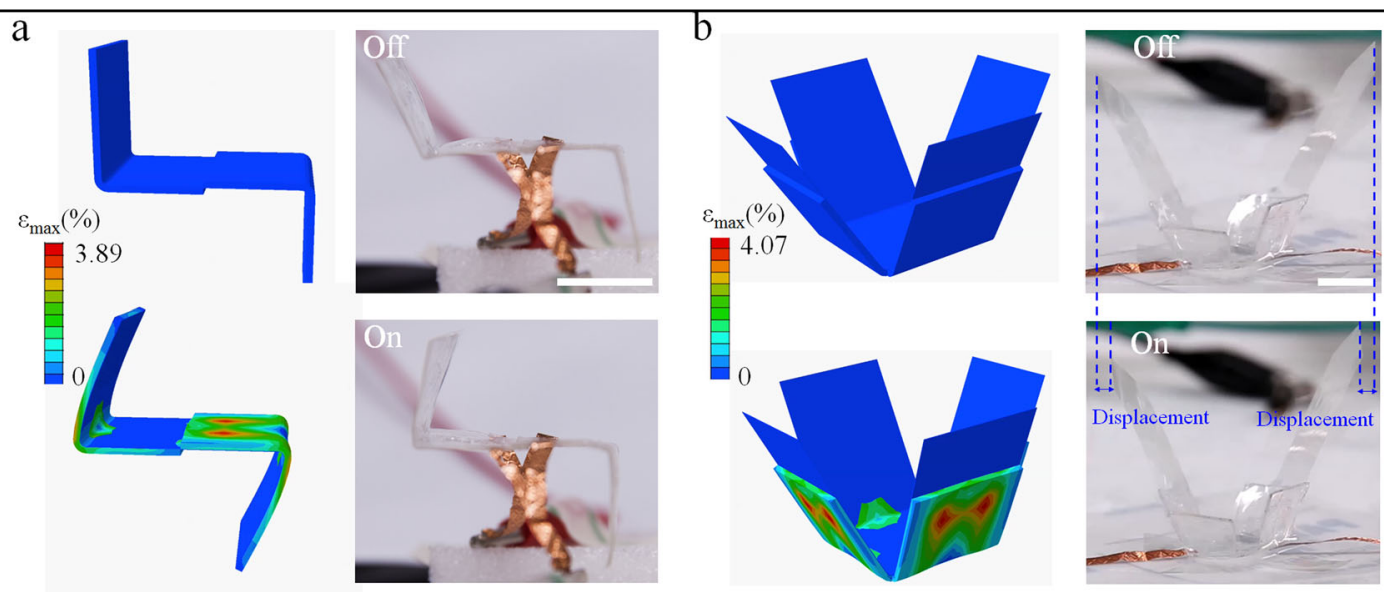

Fig. 3 The DEA devices programmed with complex 3D motions. a FEA simulations (left) and photographs (right) of the transparent actuator when the voltage is off (top) and on (bottom). b FEA simulations (left) and photographs (right) of the actuator when the voltage is off (top) and on (bottom). The scale bars in the photographs are $10 \mathrm{~mm}$. 
a

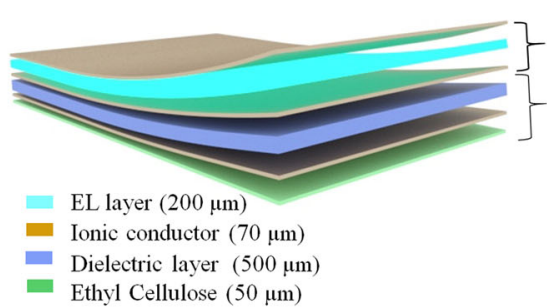

c

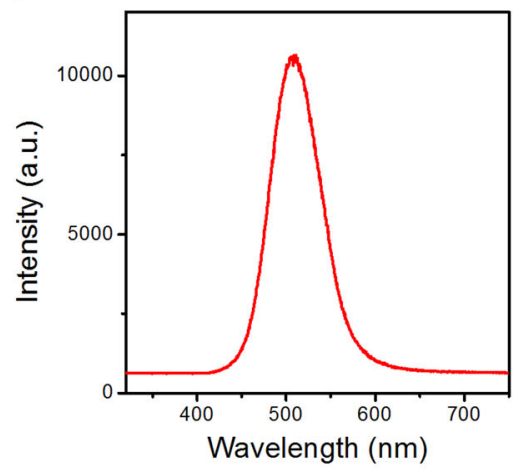

b EL layer
Transparent
Actuator

d

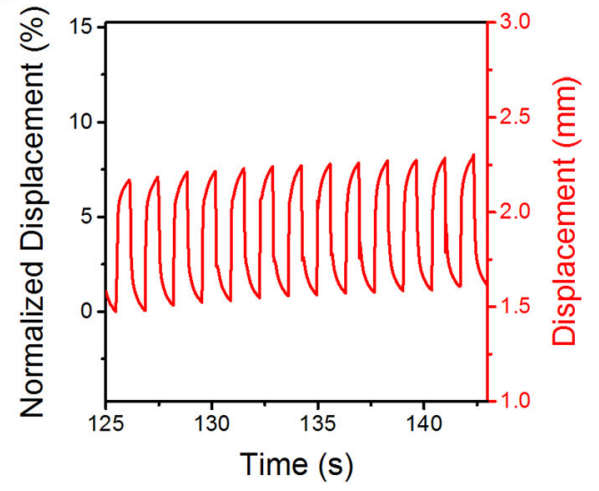

Fig. 4 The emissive flapping device. a A schematic showing the architecture of the emissive flapping device. $\mathbf{b}$ A photograph of the integrated device with light emission. The scale bar is $10 \mathrm{~mm}$. $\mathbf{c}$ The emission spectrum of the ACEL device. $\mathbf{d}$ The dynamic vertical displacement of the emissive flapping device.

\section{Discussions}

This paper has developed a simple and predictable approach to achieve transparent and programmable actuators that can be reconfigured to realize alternative shape changes. With the crease patterns, the tensile strains were symmetrically distributed with respect to the crease line under electrical bias, leading to controllable actuation behavior in the unimorph DEA devices. With the developed strategy, the conventional DEA devices could be programmed to demonstrate diverse $3 \mathrm{D}$ shape morphing based on different designs in the crease patterns. An emissive flapping device that could achieve light emission and shape morphing under electrical bias was also demonstrated to illustrate the potential applications of reconfigurable actuation in interactive electronic systems. The developed strategy highlights a promising approach to create sophisticated 3D shapes beyond the capability of conventional DEA architectures, which is believed to find wide applications in soft robotics, biological systems, volumetric displays, and camouflaging electronics. The generated force in the DEA was not as high as that of a previous report ${ }^{20}$, and it can be further improved by using multilayer structures in the DEA devices. The reconfigurable DEA devices will find promising applications in areas such as dielectric elastomer switches ${ }^{41,42}$, adaptive photonic systems ${ }^{43,44}$, and displays with configurable surface textures ${ }^{37,45}$ (Supplementary information, Fig. S7).
Further investigations on the reconfigurable actuators will focus on improving the operation frequencies of the actuator, which are significantly affected by the viscoelastic properties of the dielectric materials. Elastic polymers with less mechanical hysteresis can be exploited to tackle the challenge. The improved operation frequencies will also help improve the lift force from the actuator under flapping motions. Together with a reduction in the actuator weight, the lift force may be able to overcome the device gravity and achieve controllable flight with origami structure designs in the DEAs.

\section{Acknowledgements}

This work was financially supported by the National Research Foundation Competitive Research Programme (Award No. NRF-CRP-13-2014-02) and the NRF Investigatorship (Award No. NRF-NRFI2016-05).

\section{Conflict of interest}

The authors declare that they have no conflict of interest.

\section{Publisher's note}

Springer Nature remains neutral with regard to jurisdictional claims in published maps and institutional affiliations.

Supplementary information is available for this paper at https://doi.org/ 10.1038/s41427-019-0173-3.

Received: 10 April 2019 Revised: 3 September 2019 Accepted: 25 September 2019.

Published online: 13 December 2019 


\section{References}

1. Ko, H. \& Javey, A. Smart actuators and adhesives for reconfigurable matter. Acc. Chem. Res. 50, 691-702 (2017).

2. Yim, M. et al. Modular self-reconfigurable robot systems - challenges and opportunities for the future. IEEE Robot. Autom. Mag. 14, 43-52 (2007).

3. Gelebart, A. H., Mulder, D. J., Vantomme, G., Schenning, A. P. H. J. \& Broer, D. J. A rewritable, reprogrammable, dual light-responsive polymer actuator. Angew. Chem. Int. Ed. 56, 13436-13439 (2017).

4. de Haan, L. T., Verjans, J. M. N., Broer, D. J., Bastiaansen, C. W. M. \& Schenning, A. P. H. J. Humidity-responsive liquid crystalline polymer actuators with an asymmetry in the molecular trigger that bend, fold, and curl. J. Am. Chem. Soc. 136, 10585-10588 (2014).

5. Lahikainen, M., Zeng, H. \& Priimagi, A. Reconfigurable photoactuator through synergistic use of photochemical and photothermal effects. Nat. Commun. 9 4148 (2018).

6. Pelrine, R., Kornbluh, R., Pei, Q. B. \& Joseph, J. High-speed electrically actuated elastomers with strain greater than 100\%. Science $\mathbf{2 8 7}, \mathbf{8 3 6}-839$ (2000).

7. Brochu, P. \& Pei, Q. B. Advances in dielectric elastomers for actuators and artificial muscles. Macromol. Rapid Commun. 31, 10-36 (2010).

8. Acome, E. et al. Hydraulically amplified self-healing electrostatic actuators with muscle-like performance. Science 359, 61-65 (2018).

9. Xu, C. Y., Stiubianu, G. T. \& Gorodetsky, A. A. Adaptive infrared-reflecting systems inspired by cephalopods. Science 359, 1495-1500 (2018).

10. Zhao, X. H. \& Suo, Z. G. Theory of dielectric elastomers capable of giant deformation of actuation. Phys. Rev. Lett. 104, 178302 (2010).

11. Marette, A. et al. Flexible zinc-tin oxide thin film transistors operating at $1 \mathrm{kV}$ for integrated switching of dielectric elastomer actuators arrays. Adv. Mater. 29, 1700880 (2017)

12. Hines, L., Petersen, K. \& Sitti, M. Inflated soft actuators with reversible stable deformations. Adv. Mater. 28, 3690-3696 (2016).

13. Keplinger, C., Li, T. F., Baumgartner, R., Suo, Z. G. \& Bauer, S. Harnessing snapthrough instability in soft dielectrics to achieve giant voltage-triggered deformation. Soft Matter 8, 285-288 (2012).

14. Godaba, H., Foo, C. C., Zhang, Z. Q., Khoo, B. C. \& Zhu, J. Giant voltage-induced deformation of a dielectric elastomer under a constant pressure. Appl. Phys. Lett. 105, 112901 (2014)

15. Boys, H., Frediani, G., Poslad, S., Busfield, J. \& Carpi, F. A dielectric elastomer actuator-based tactile display for multiple fingertip interaction with virtual soft bodies. In Presented at the SPIE Smart Structures and Materials + Nondestructive Evaluation and Health Monitoring, Portland, Oregon, Society of Photo-Optical Instrumentation Engineers (SPIE), 2017.

16. Koo, I. et al. Wearable tactile display based on soft actuator. In Proc. 2006 IEEE International Conference on Robotics and Automation (ICRA). IEEE, Vol. 1-10, 2220-2225 (2006).

17. Koo, I. M. et al. Development of soft-actuator-based wearable tactile display. IEEE Trans. Robot. 24, 549-558 (2008).

18. Haghiashtiani, G., Habtour, E., Park, S. H., Gardea, F. \& McAlpine, M. C. 3D printed electrically-driven soft actuators. Extreme Mech. Lett. 21, 1-8 (2018).

19. Shian, S., Bertoldi, K. \& Clarke, D. R. Dielectric elastomer based "grippers" for soft robotics. Adv. Mater. 27, 6814-6819 (2015).

20. Duduta, M., Wood, R. J. \& Clarke, D. R. Multilayer dielectric elastomers for fast, programmable actuation without prestretch. Adv. Mater. 28, 8058-8063 (2016).

21. Lau, G. K., Goh, S. C. K. \& Shiau, L. L. Dielectric elastomer unimorph using flexible electrodes of electrolessly deposited (ELD) silver. Sens. Actuators A Phys. 169, 234-241 (2011)
22. Araromi, O. A. \& Burgess, S. C. A finite element approach for modelling multilayer unimorph dielectric elastomer actuators with inhomogeneous layer geometry. Smart Mater. Struct. 21, 032001 (2012).

23. Zhang, K. et al. Origami silicon optoelectronics for hemispherical electronic eye systems. Nat. Commun. 8, 1782 (2017).

24. Miskin, M. Z. et al. Graphene-based bimorphs for micron-sized, autonomous origami machines. Proc. Natl Acad. Sci. USA 115, 466-470 (2018).

25. Rus, D. \& Tolley, M. T. Design, fabrication and control of origami robots. Nat. Rev. Mater. 3, 101-112 (2018).

26. Faber, J. A., Arrieta, A. F. \& Studart, A. R. Bioinspired spring origami. Science 359 1386-1391 (2018)

27. Mahadevan, L. \& Rica, S. Self-organized origami. Science 307, 1740-1740 (2005).

28. Li, S. G., Vogt, D. M., Rus, D. \& Wood, R. J. Fluid-driven origami-inspired artificial muscles. Proc. Natl Acad. Sci. USA 114, 13132-13137 (2017).

29. Martinez, R. V., Fish, C. R., Chen, X. \& Whitesides, G. M. Elastomeric origami: programmable paper-elastomer composites as pneumatic actuators. Adv. Funct. Mater. 22, 1376-1384 (2012).

30. Jin, B. J. et al. Programming a crystalline shape memory polymer network with thermo- and photo-reversible bonds toward a single-component soft robot. Sci. Adv. 4, eaao3865 (2018).

31. Xiong, J. Q. et al. A deformable and highly robust ethyl cellulose transparent conductor with a scalable silver nanowires bundle micromesh. Adv. Mater. 30, 1802803 (2018).

32. Wang, J. et al. Extremely stretchable electroluminescent devices with ionic conductors. Adv. Mater. 28, 4490-4496 (2016)

33. Keplinger, C. et al. Stretchable, transparent, ionic conductors. Science 341, 984-987 (2013).

34. Sun, J. Y., Keplinger, C., Whitesides, G. M. \& Suo, Z. G. Ionic skin. Adv. Mater. 26, 7608-7614 (2014).

35. Wang, J., Yan, C., Chee, K. J. \& Lee, P. S. Highly stretchable and self-deformable alternating current electroluminescent devices. Adv. Mater. 27, 2876-2882 (2015).

36. Wang, J. X., Lin, M. F., Park, S. \& Lee, P. S. Deformable conductors for humanmachine interface. Mater. Today 21, 508-526 (2018).

37. Pikul, J. H. et al. Stretchable surfaces with programmable 3D texture morphing for synthetic camouflaging skins. Science 358, 210-214 (2017).

38. Trimmer, B. A. et al. Nitric oxide and the control of firefly flashing. Science $\mathbf{2 9 2}$, 2486-2488 (2001)

39. Yang, C. H., Chen, B. H., Zhou, J. X., Chen, Y. M. \& Suo, Z. G. Electroluminescence of giant stretchability. Adv. Mater. 28, 4480-4484 (2016).

40. Larson, C. et al. Highly stretchable electroluminescent skin for optical signaling and tactile sensing. Science 351, 1071-1074 (2016).

41. Anderson, I. A., Gisby, T. A., McKay, T. G., O'Brien, B. M. \& Calius, E. P. Multifunctional dielectric elastomer artificial muscles for soft and smart machines. J. Appl. Phys. 112, 041101 (2012).

42. McKay, T. G., O'Brien, B. M., Calius, E. P. \& Anderson, I. A. Soft generators using dielectric elastomers. Appl. Phys. Lett. 98, 142903-142903-3 (2011).

43. Kornbluh, R. D. et al. Shape control of large lightweight mirrors with dielectric elastomer actuation. In Proc. Smart Structures and Materials, 2003: Electroactive Polymer Actuators and Devices (EAPAD), Society of Photo-Optical Instrumentation Engineers (SPIE), Vol. 5051, 143-158 (2003).

44. Heimann, M., Schröder, H., Marx, S. \& Lang, K. -D. Dielectric elastomer actuators for adaptive photonic microsystems. In Proc. Organic Photonic Materials and Devices XV, Society of Photo-Optical Instrumentation Engineers (SPIE), Vol. 8622, 862209 (2013)

45. Wang, J. X., Yan, C. Y., Chee, K. J. \& Lee, P. S. Highly stretchable and selfdeformable alternating current electroluminescent devices. Adv. Mater. 27, 2876-2882 (2015) 